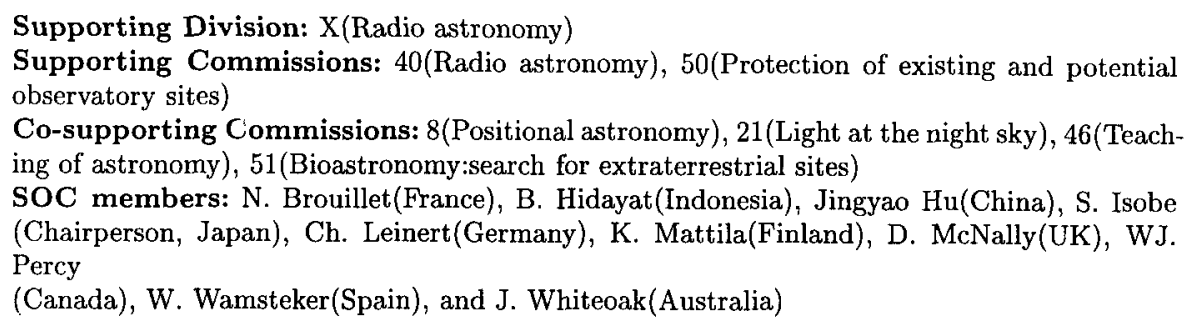

Held on August 21 and 22, 1997, during the XIIIrd General Assembly in Kyoto.

In these decades astronomy has been developing very much because observations in optical, radio, and the other wavelength domains as well as neutrino, cosmic ray, and in-situ observations have been extensively carried out collaborating with theoretical works. However, it is also true that astronomical observational conditions have become worse and worse during these same period. Astronomical observers are suffering light pollution, radio interference, and space debris. IAU had realized these problems and set up a commission to deal these matter in 1978 : that is the Commission 50 "Protection and identification of existing and potential observational site. The Commission organized the IAU Colloquium No. 112" (D. Crowford 1991) in Washington D. C, USA, from 13 to 16 August 1988, and extensively discussed the matters. Some number of astronomers worked so hard to reduce the problems by contacting and collaborating with the other organizations such as CIE, COSPAR, URSI, ITU, IAD, and UNESCO, UN. One of these activities was a joint meeting of UNESCO, ICSU, IAU, and CSR held in Paris from June 30 to July 21992 , that is "Adverse Environmental Impact on Astronomy" (D. McNally 1994). However, since most of the observational astronomers have been busy to work out their own observations, the problems have still become worse.

The JD5 is a meeting inside the IAU. The main purpose of this JD is to know present situations of different interferences, to find ways to reduce the problems, and most importantly to make many IAU members realize the problems. Fortunately, we could have a talk by L. Woltjer, the President of the IAU, relating investments of astronomy and those loss by the problems dealed in this JD5. This is an important point which we, astronomers, should always think about Astronomy uses some billion US dollars per year and our loss becomes some fractions of this value.

We had 23 talks including the President, 9 paster papers, and nearly 200 audiences who attended at least some session(s). Therefore, the JD5 was successful with many discussions.

To approach public people, different organizations, different governments, and UN, on the problems, there are many ways, but it is inevitably necessary for us, astronomers, to do much efforts. Astronomers actively working on the problems gave reports during the JD5, and the followings are short summaries of their talks. Those full papers describing in much detail will be published in the other book (Isobe and Hirayama 1998). It is highly expected that all the IAU members start to consider the problems which may kill astronomical observations.

Syuzo Isobe (Chairman of JD5)

\title{
1. ADVERSE ENVIRONMENTAL IMPACT ON ASTRONOMY
}

D. McNally (University of London Observatory) 
This paper sets out to urge the quantification of adverse environmental impact on astronomy (AEI). Up to the present AEI has been treated anecdotally. This is not a criticism - the demonstrations have been compelling, particularly so in the case of light pollution. However, we must now move from demonstration to effective action. That effective action will impact the legitimate activities of others. Therefore astronomy will have to present evidence capable of convincing not just those sympathetic to the astronomical community, but the community in general that astronomy has a legitimate grievance and that the redress being sought will be effective in improving astronomical observing condition.

In order to be convincing we must quantify. We must be able to state, for example, what background levels of light pollution are, what the normal range of the level is and how the level is changing from year to year. All claimed adverse impact should have some measure to express the real nature of the impact. Only when such measures are in place can we argue for legal protection.

The problems to be faced in quantification must not be minimized. The problems are considerable and each adverse impact has its own unique set. Some of the problems are outlined in the paper. Unfortunately time is not on our side. Every year sees a rebirth of the concept of advertising from space in one form or another. Sooner or later, unless we are supremely vigilant, a proposal will be accepted and the consequences will be disastrous for our science. Hence the thrust of the EC Resolution before this General Assembly.

There is now great investment in astronomy as a science. That investment has produced some of the most exciting astronomy in all history. One only has to think of the spectacular discoveries of the HST, the advance in basic astrometric data represented by HIPPARCOS, and the unparalleled spectroscopic data base from IUE. We are currently looking forward to the fruits of the $10 \mathrm{~m}$ generation optical telescopes and sustained adventures in radio astronomy. Yet that tantalisingly brilliant future is at risk.

Unfortunately the interests of astronomy are now in conflict with other human activities. Astronomy has retreated to its mountains, deserts and into space. It can no longer retreat-there is nowhere left to go. The other competing interests are well heeled profitable and will claim enormous benefits for society. Some of the benefits are real but not all. We as astronomers will have to come to terms with the rest of society- but by the same token, the rest of society will have to come to terms with us - if astronomy is to survive as a major science. It would be a great disaster for the whole of science were the science of astronomy to be curtailed by the pollution produced terrestrially. Loss of investment would be disaster, loss of cosmic perspective would be a catastrophe.

\section{IMPACT ON RADIO ASTRONOMY}

\section{Morimoto (Space Science Course, Kagoshima University)}

The radio sky has continuously supplied "exotic objects" to astronomy, such as quasars, pulsars etc. After a while these objects proved to be important constituents of the Universe and provided a new look of the Universe.

Cosmic Background Radiation is the remnant of the blackbody radiation of Universe when it was cooled to about $3000 \mathrm{k}$. Density of quasars is higher in the distant part of the Universe and proves the Universe was more crowded in the early stage.

Spectral line emission from molecules formed in the interstellar gas clouds shows processes of star formation in the Galaxy and galaxies.

Technology in radio telescope in also continuously in a rapid progress. Recent success of HALUCA satellite and VSOP has opened a new era of high resolution radio imaging through space VLBI. Accuracy of source position measurements is approaching 1 microarcsec as level and trigonometry will soon cover whole Galaxy and beyond.

These are only a small portion of achievements of radio astronomy, but prove that it continucs to contribute important parts of the human knowledge of the Universe.

On the other hand, this exciting field of science is critically endangered by the more and more: intense usage of radio spectrum for communication and other purposes.

Frequencies below $\sim 300 \mathrm{MHz}$ (m-waves and longer)

Low frequency radio emission represents low density plasmas in Universe, which is clifficult to observe by other means. Examples are outer corona of the sun, envelope of the Galaxy and 